\title{
Impact of Large Distributed Solar PV Generation on Distribution Voltage Control
}

\author{
Miroslav Begovic \\ Texas A\&M University Texas A\&M University \\ Shipra Mohan \\ begovic@tamu.edu peerzada@tamu.edu \\ IIT, Gandhinagar \\ shipra.mohan@iitgn.ac.in \\ Robert Balog \\ Texas A\&M University, Qatar \\ robert.balog@qatar.tamu.edu
}

Wesam Rohouma

Texas A\&M University, Qatar

\begin{abstract}
The emergence of distributed generation in the low voltage distribution networks has led to new challenges in the regulation of feeder voltages. Of particular significance is the variability associated with the photovoltaic power and its impact on the operation of some of the mechanically switching voltage regulating equipment such as On-load Tap Changers (OLTCS) and switchable capacitor banks. This article attempts to study the effects of increased penetration of distributed generation, in particular photovoltaic power and a sustained load buildup on the operational activity of voltage regulators placed on a radial distribution feeder. Actual feeder load profile and high frequency solar irradiance data has been used with varying levels of $P V$ penetration during the time period which spans an entire year. With the inclusion of some justifiable assumptions, it is concluded that the increased penetration of photo voltaic power adversely affects the operational lifetime of voltage regulating equipment.

Keywords: renewable generation, solar $P V$ integration, voltage control in distribution networks.
\end{abstract}

\section{Introduction}

The recent expansion of the distributed energy resources, particularly photovoltaic power, in the distribution networks, has resulted in several voltage regulation issues. With penetration projected to only increase in the future, network operators will have to contend with an increasing surge in the range of problems concerned with the regulation of voltage and power on distribution feeders. For example, in San Diego Gas and Electric distribution territory, PV installations accounted for $617 \mathrm{MW}$ of peak load from 93000 installations at the end of June 2016. In August of 2013, these numbers were $175 \mathrm{MW}$ from $24000 \mathrm{PV}$ installations. This represents a substantial increase in the PV penetration over the course of roughly three years. The high penetration of PV can be attributed in large measure to the policies pursued by the federal as well many of the state governments with an increasing concern for climate change and declining PV system costs among other factors.

The fundamental concern about the photovoltaic generation is the intermittency involved which when combined with other factors such as a fluctuating load profile can induce irregularities not only in the voltage on the distribution feeder but also in the operational activity of several electro-mechanical devices which are designed and placed on the feeder for the purpose of voltage regulation. This places pressure on the distribution utilities which are obligated under the ANSI standards to provide voltage within a dead band of $\pm 5 \mathrm{~V}$ from the nominal distribution voltage. While the impact of such distribution generation in general and photovoltaic penetration, in particular on the voltage profile in the low voltage networks has been documented in a number of studies, the impact of such solar intermittency on the operational life cycle of voltage regulating equipment has not been thoroughly investigated [1-2]. Some of the studies performed on the occurrences of tap changing operations either didn't utilize high frequency solar irradiance data or focused on such operational irregularities over an inadequate period of time. It is important to mention that both the factors, the use of high frequency solar irradiance and an adequate period of interest are very important in assessing such induced variabilities in the operational activity of such electro-mechanical devises. In [3], the impact of PV penetration on a distribution system with a $20 \mathrm{MVA}, 69 \mathrm{kV} / 12,47 \mathrm{kV}$, $\Delta-\mathrm{Y}$ connected transformer, serving two residential feeders was demonstrated. It is shown that a $20 \% \mathrm{PV}$ penetration amounting to $4 \mathrm{MWs}$ can increases the number of tap changing operations four times compared with $0 \%$ PV penetration. However, the study was performed on global horizontal irradiance data of 1-minute interval for a period of three days in the summer. As such the study ignores the effect of tilt angle and the azimuth angle of the solar PV panel on the PV output. Furthermore, the study assumes a 
lumped load model at the transformer secondary bus, thus ignoring the voltage drop along the feeder.

In [4], a yearlong of PV data of 1-minute interval was used on a model of a residential network consisting of 60 houses. A $33 \mathrm{kV} / 11 \mathrm{kV}$ transformer is used to step down the upstream voltage which is further stepped down to $415 \mathrm{~V}$. The PV data used for modelling has a resolution of 1 minute and accounts for 1.22 MWp in a system with $12.5 \mathrm{MW}$ base load and $22 \mathrm{MW}$ peak load with an assumed average nominal load of each household to be $3 \mathrm{~kW}$, implying a $10 \%$ or less PV penetration. It is shown that with increased variability of the PV output, the number of tap changing operations also increase and the operations saturate at a PV penetration of more than $90 \%$. The dependence of the solar irradiance on the tilt angle of the solar panel and the azimuth however is not specified. In [5], a characterization of the high frequency solar variability based on the ramp rate distributions at ten different locations across the United States is provided. Weekly simulations with a time resolution of $1 \mathrm{~s}$ are carried on a $12 \mathrm{kV}$ agricultural feeder and the impact on the voltage regulator operations is studied. Global horizontal irradiance is used to compute the power output of a 3 MW single axis tracking PV power plant. However, since the study is carried out on a weekly basis and hence is inadequate for capturing the seasonal shifts of the PV output. Moreover, the study ignores the impact of scattered PV installations across the feeder. In [6], the study tries to assess the variability in the load and solar irradiance from a frequency domain perspective. The study outlines the impact of solar irradiance on the feeder load and the modifications caused to the net load as seen by the utility.

Several methods have also been proposed to mitigate the effect of the solar variability on the operation of voltage regulating equipment. The use of on-site battery storage is presented in [7]. In [8], an optimization problem is formulated to minimize the operation of voltage regulating equipment by dispatching an optimal reactive power control strategy based on the load and the irradiance forecast. The method is tested on an $11 \mathrm{kV}$ network with 95 buses equipped with two PV plants with a combined output of $2 \mathrm{MW}$, operating at $0.95 \mathrm{lead} / \mathrm{lag}$ power factor. The month-long solar data has a time resolution of 30 seconds coupled with a half hourly load profile. Reference [9], tested the performance of the transposition models that attempt to estimate the Global Tilt Irradiance from the measured high frequency horizontal irradiance data sets and found that the uncertainty associated with the tilted irradiance is roughly $3 \%$ for axis global solar panels and approximately $5 \%$ for panels with fixed tilts.
This article attempts to contribute to the already accumulated knowledge of the impact of high frequency solar variability on the electro-mechanical devices in the distribution feeders. The final objective is to estimate the total number of annual operations of such devices based on an actual IEEE test feeder with a given solar irradiance profile and a load profile. For the solar irradiance, actual values of Global Horizontal Irradiance (GHI), Direct Normal Irradiance (DNI) and Diffused Horizontal Irradiance (DHI) with a time resolution of 1 minute are used and converted to the Global Tilt Irradiance (GTI), with an assumed tilt angle and a given array azimuth. The load profile is sampled hourly for an entire year with real residential load data and coupled with the high frequency tilted irradiance, the PV output of a number of scattered installations across the test feeder is estimated. It is found that an increase in the penetration of $\mathrm{PV}$ on the feeder has an adverse impact on the operational activity of voltage regulators.

\section{High Frequency Data}

To properly account for the impact of photovoltaic power on the distribution grids, in particular the operation of the regulator tap changers, it is imperative to sample the incoming solar radiation at higher frequencies. Since the time constant of tap changers is typically shorter than 1-minute, high frequency solar data will better approximate the fast variabilities of the $\mathrm{PV}$ output power and the consequent reaction of the tap changers. The lack of availability of the global tilted irradiance makes it necessary to use some transposition models on the horizontal irradiance which depends on the tilt angle of the PV array as well as the direction in which the array is facing. Consequently, the net PV output has a relational dependence of the angle of tilt and the array azimuth. The data for the GHI, DNI, DHI, the solar azimuth and the solar zenith angle has been made public by the National Renewable Energy Laboratory. Such data are specific to a particular location with a given elevation. Since this study is performed on an actual distribution feeder located in Arizona, the solar data for the southwest region of the United States was used in the analysis. The use of actual local solar data with a high time resolution coupled with actual local load profile data, on an actual distribution feeder helps to validate the results of this analysis and keeps the study grounded in reality.

A major chunk of the previous effort in this direction has made use of either low frequency GHI or high frequency GHI. However, horizontal irradiance can't accurately represent the PV power output as it has a direct dependence on the panel tit angle as well 
as the array azimuth. According to an NREL study, a common rule of thumb for the tilt angle for fixed arrays is that the for maximum power output, the tilt angle should roughly be equal to the latitude of the system location. Of course, given the change in the sun's apparent position in the sky throughout the year, the optimal tilt angle and the array azimuth also changes. However, for the purposes of this study and at first approximation, the tilt angle of the array is assumed to be constant throughout the year and is equal to the latitude of Phoenix, Arizona which is 33.3 degrees N. For the array azimuth, all the solar PV installations are assumed to be facing south and since the system location is in the northern hemisphere, the array azimuth is taken to be 180 degrees. Given the measured data sets for the GHI, DNI and DHI, the global irradiance for a panel with a tilt angle of ' $\beta^{\prime}$, is given by the expression [9]

$$
E_{\beta}=E_{D N I} \cos \theta+E_{D H I} R_{d}+\rho E_{G H I} R_{r}
$$

Where $E_{D N I}$ is the direct normal irradiance, $E_{D H I}$ is the diffused horizontal irradiance, $E_{G H I}$ is the global horizontal irradiance in watts $/ \mathrm{m}^{2}$. The angle $\theta$ is the angle of incidence of the sun's rays on the plane of the solar array, $R_{d}$ is the diffused transposition factor, $\rho$ is the foreground's albedo and $R_{r}$ is the diffusion reflection factor for the reflection from the ground. Clearly an accurate estimation of the tilted irradiance will require an accurate measurement of the irradiances on the right hand side of equation (1) along with a realistic approximation of the factors, $R_{d}, R_{r}$ and $\rho$. The angle of incidence is given by the expression

$\theta=\cos ^{-1}\left[\cos Z \cos \beta+\sin Z \sin \beta \cos \left(A_{\text {sol }}-A_{p v}\right)\right]$ Where $Z$ is the solar zenith angle, $\beta$ is the assumed tilt angle of the PV array, $A_{\text {sol }}$ is the solar azimuth angle and $A_{p v}$ is the array azimuth angle. With the changes in the solar zenith angle and the solar azimuth, the angle of incidence varies throughout the day with the change in the apparent position of the sun. The angle of incidence is calculated at every new time step to obtain the tilted irradiance. The irradiances on the right hand side of equation (1) also share an interrelationship given by

$$
G H I=D N I \cos (Z)+D H I
$$

$Z$ represents the solar zenith angle. This measurement of the zenith angle is available in the NREL database and does not need to be calculated separately. For studying the impact of PV penetration and as a first approximation study it is reasonable to make some justifiable assumptions about some ambient conditions which would simplify the modelling of transposition factor, reflection factor and the albedo. For the reflection factor, the ground reflection is assumed to ideally isotropic and the diffused irradiance is assumed to be constant over the whole sky hemisphere. With the help of these simplifying assumptions, $R_{d}$ and $R_{r}$ can be modelled as

$$
\begin{aligned}
& R_{d}=\frac{1+\cos \beta}{2} \\
& R_{r}=\frac{1-\cos \beta}{2}
\end{aligned}
$$

For the solar albedo factor, $\rho$ which defines the ratio of irradiance reflected to the irradiance received by the surface, a value of 0.2 is widely accepted with an upper threshold of 0.5 . In this study, $\rho$ is taken as 0.2 . Reference [9] carries a more detailed explanation behind some of the assumptions made here and also a quantification of some performance issues is presented under such assumptions. For the purposes of our study, the aforementioned model seems to be sufficiently robust in carrying out the distribution system analysis.

By considering all the variables, albeit approximately, a more robust measurement of the variability associated with the solar PV output is possible. The data sets for GHI, DNI and DHI used in this study have a time resolution 1 minute with varying solar azimuth and the solar zenith angle. The preference of solar data sampled at a higher frequency can be explained by observing Figure 1 and Figure 2 .

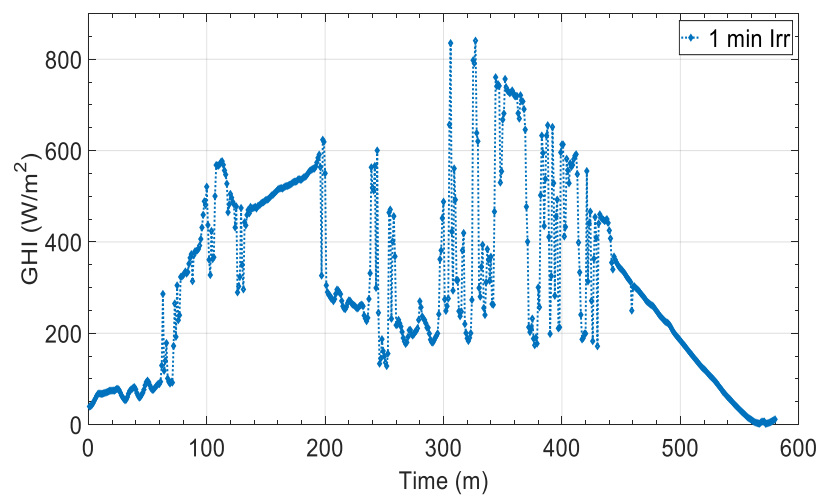

Figure 1. GHI Profile with a time resolution of 1 minute for one operational day.

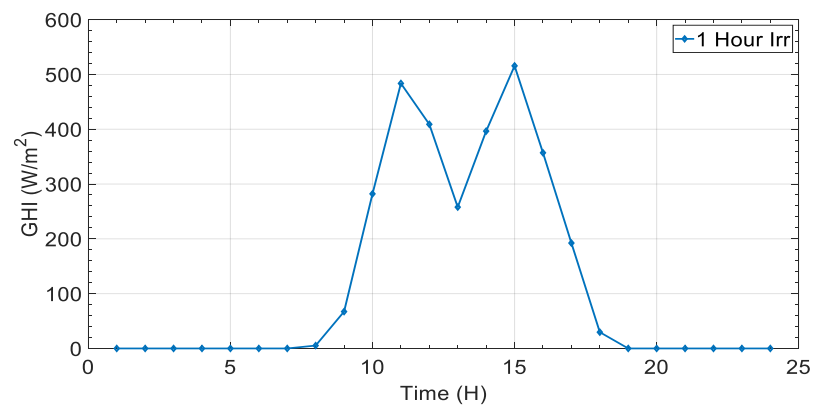

Figure 2. GHI Profile with a time resolution of 1 hour for one operational day. 
The irradiance profile presented in Figure 2 may be suitable for some energy calculations but it is clearly inadequate for assessing the dynamic performance of a PV module as a result of cloud induced transients. Tap changing devices and other electro-mechanical devices on the distribution feeder will react quickly to the transitory rises and drops in the irradiance which are completely ignored in lower frequency measurements. The irradiance profile in Figure 1 and Figure 2 are presented for Phoenix area on the day of January 16, 2012.

\section{Test System Description and Modelling}

To quantify the effects of high PV penetration on distribution feeders, IEEE 34 node feeder was chosen in this study owing to its large radial length and high voltage imbalance. It represents a typical rural distribution network with single and three phase laterals which can incorporate distributed generation. Voltage compensation is provided by three phase online capacitor banks and two voltage regulators. The total feeder length is 94 kilometers and a $69 / 24.9 \mathrm{kV}$ transformer is used to step down the primary voltage. Actual phase impedance values are used to model the three phase and the single-phase overhead lines. The substation is rated at $2500 \mathrm{kVA}$ and the system base active load is $1769 \mathrm{~kW}$. This includes a combination of the spot loads and the distributed loads. The spot loads make up $1077 \mathrm{~kW}$ of active load and $677 \mathrm{kVAr}$ of reactive load on the system while the distributed loads comprise of $722 \mathrm{~kW}$ of base active load and 367 $\mathrm{kVAr}$ of base reactive load. In addition to the main substation transformer, there is also an in-line step down transformer connected between buses 832 and 888 . The secondary voltage of this transformer is 4.16 $\mathrm{kV}$. The test system comprises of two three phase capacitor banks connected to buses 844 and 848 . The reactive injection from capacitors is used for power factor correction of the source. The one-line diagram of the test system is given in Figure 3.

Voltage Regular 1 is located in between buses 814 and 850 and provides voltage regulation on the secondary side with a dead band of $2.0 \mathrm{~V}$, whereas the second regulator connects buses 832 and 852 with the same specifications for the dead band. Three phase capacitor banks rated at $300 \mathrm{kVAr}$ and $450 \mathrm{kVAr}$ are connected at buses 844 and 848 and operate at a line to line voltage of $24.9 \mathrm{kV}$. The ratings of the capacitors are decided by the amount of reactive power delivered by the substation transformer. The lines in the system are three phase overhead and single-phase overhead with varying degree of imbalance. Loads on the feeder are modelled as three phase (balanced or unbalanced), spot or distributed (single phase or three phase).

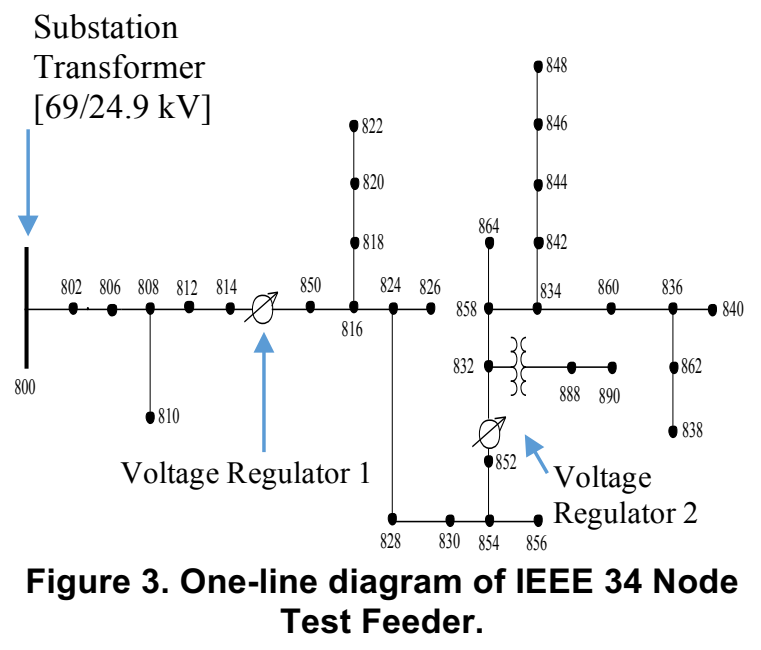

Three phase loads can either be connected in wye or delta while single phase loads are either connected line to ground or line to line. A variety of models are used for modelling loads which included constant $\mathrm{P}$, constant PQ, constant Z, constant I and exponential loads. The various components of the test system are modelled in OpenDSS and the data processing is done in MATLAB. OpenDSS models an electrical component of a circuit by creating a primitive admittance matrix of the element and the system admittance is obtained by combining all the elemental $\mathrm{Y}$ matrices.

The substation transformer is modelled as three phase 2 winding transformer with the operating primary voltage of $69 \mathrm{kV}$. There are 32 tap positions with uniform leakage impedance on the LV and HV side. The tap positions need to take care of the daily fluctuations in the voltage. They allow the ratio to vary in the range of $\pm 10 \%$ with each tap change resulting in $0.00625 \mathrm{p} . \mathrm{u}$ change in voltage. The two voltage regulators were modelled as single-phase autotransformers. One possible restriction in OpenDSS is that all conductors at a circuit element terminal must be connected to the same bus. This restriction however does not apply to nodes in a bus. OpenDSS allows the use of a regulator control device which monitors the tap positions on the winding of the autotransformer it is placed on. The two regulators are connected in wye configuration with the tap position of winding 2 being controlled by the regulator control element. The PT ratio is set as 120 and the CT ratio is set as 100 . The desired voltage is set as $122 \mathrm{~V}$ and a band of $2 \mathrm{~V}$ is selected.

OpenDSS utilizes a combined model for the PV array and the inverter. The model is valid for a step size of greater than 1 second. The model consists of a 
PV array, the output of which is fed to an inverter with a pre-defined efficiency curve. The efficiency of the inverter will depend on the $\mathrm{V}_{\mathrm{MPP}}$, which refers to the voltage at the maximum power point. However, OpenDSS accepts only one efficiency curve among a family of possible curves. As seen from the rest of the circuit, a PV system appears a power conversion element, similar to a generator or a load, which generates or consumes power in accordance to some function. The PV active power output depends on the value of irradiance specified, the temperature and rated peak power $\mathrm{P}_{\mathrm{mpp}}$, at the maximum power point. $\mathrm{P}_{\mathrm{mpp}}$, specified by the user is defined at standard test conditions, which are an irradiance of $1 \mathrm{~kW} / \mathrm{m}^{2}$ and a temperature of $25 \mathrm{C}$. The reactive power is specified separately either as fixed power factor or fixed $\mathrm{kVAr}$ values. Unity power factor control is implemented in the simulation. The panel output is calculated by:

$$
P_{k W}(t)=P_{M P P}(S T C) \times \operatorname{Ir} r_{\text {base }} \times \operatorname{Irr}(t) \times T_{f}(t)
$$

Where $P_{k W}(t)$ is the output from the PV panel at a given time, $P_{M P P}(S T C)$ is the power at the maximum power point defined under standard test conditions $\left(1 \mathrm{~kW} / \mathrm{m}^{2}\right.$ irradiance and $25 \mathrm{C}$ temperature), $I r r_{\text {base }}$ is the base value of irradiance usually taken to be $1 \mathrm{kw} / \mathrm{m}^{2}, \operatorname{Irr}(t)$ is the current irradiance and $T_{f}(t)$ is the current temperature factor which is interpolated from the temperature curve defined by the user. The panel output is multiplied by the efficiency of the inverter to yield the PV output power which is fed to the feeder.

$$
P_{P V}(t)=\eta_{\text {inverter }} P_{k W}(t)
$$

In order to study the impact of PV variability on the voltage regulating equipment, following assumptions were made to simplify the calculation process.

- For the transposition model to obtain the global tilted irradiance, the foreground albedo factor was assumed to 0.2 which is a standard practice for solar PV designs. Additionally, the ground reflection is assumed to be ideally isotropic and diffused irradiance is assumed to be constant over the whole sky hemisphere. This assumption yields $R_{d}=0.917$ and $R_{r}=0.0852$.

- The effect of nearby shading from buildings and the atmospheric scattering on the PV output is not considered.

- $\quad$ Finally, all the PV installations were assumed to receive the same solar irradiance and all the loads were subjected to a common load profile. This assumption was made because of the difficulties involved in getting individual load profiles and separate solar irradiance profile for a single feeder.

\section{Simulation Results}

The test feeder was simulated using a snap shot simulation as well as a quasi-time series simulation. The snap shot simulations are important to assess some feeder properties and look for voltage violations along the length of the feeder. For the snap shot simulation, three different system conditions were studied. In the first case, the feeder was stripped of any voltage compensation. In the second case, capacitor compensation was provided to improve the voltage profile and the source power factor. It was seen that the reactive power injection by the capacitor banks corrected the voltage at some of the buses on the feeder and the source power factor was also improved. In the third case of snap shot simulation, voltage regulators were connected at locations which exhibited depressed voltages even with capacitor compensation. In the final case of snap shot simulations, an annual load growth of $3 \%$ was assumed on the feeder and the feeder voltage and the voltage regular tap positions were simulated at the end of the tenth year. For the lack of space, the results for the final two cases are presented here since the main focus of this study is the operational irregularities observed in the tap changing devices due to fluctuations in the system load and the variable generation induced by PV penetration.

\subsection{Snap Shot Simulations}

For correcting the low voltage problems, voltage regulators modelled as single-phase autotransformers are installed along the feeder. Since the minimum acceptable voltage on the feeder is 0.95 p.u, the voltage profile of the buses is scanned and the first bus, as measured from the substation, where the voltage violation is observed is chosen for the placement of the voltage regulator. This correction is be made by installing a voltage regulator at Bus 814 . With the regulator installed and the tap settings determined by the Regulator Control device, the voltage profile is greatly improved. The voltages at Bus $814 \mathrm{r}$ seem to satisfy the voltage constraint. However, the voltage at bus 890 is still in violation of the first constraint. To raise the voltage in the $888-890$ lateral, it is necessary to boost the primary voltage of the inline transformer $24.9 / 4.16 \mathrm{kV}$. To accomplish this, the compensator settings are made $\mathrm{R}=2.5$ and $\mathrm{X}=1.5$ and the desired voltage is set at $122 \mathrm{~V}$ on a $120 \mathrm{~V}$ scale. This installation should be able to boost the primary side voltage of the inline transformer by causing the regulator to adjust taps and thereby increasing the depressed voltages all along the downstream feeder. 
With capacitors and voltage regulators on the feeder, the overall system losses which includes the line losses and the transformer losses have been substantially reduced from the initial value of $20.15 \%$ in the uncompensated case and $16.8 \%$ in the case where only capacitors were deployed on the feeder.

Table 1. Case Results Summary

\begin{tabular}{|l|l|}
\hline $\begin{array}{l}\text { Active Power Supplied by } \\
\text { Source }\end{array}$ & $2039 \mathrm{~kW}$ \\
\hline $\begin{array}{l}\text { Reactive Power Supplied by } \\
\text { Source }\end{array}$ & $281 \mathrm{kVAr}$ \\
\hline Source Power Factor & 0.9906 \\
\hline Line Losses & $262.2 \mathrm{~kW}$ \\
\hline Transformer Losses & $10 \mathrm{~kW}$ \\
\hline Total Losses & $272.3 \mathrm{~kW}$ \\
\hline Total Load Power & $1767.6 \mathrm{~kW}$ \\
\hline Percent Loss for Circuit & $15.4 \%$ \\
\hline
\end{tabular}

Table 2. Regulator Tap Positions at Base Year

\begin{tabular}{|l|l|l|l|l|}
\hline Name & Tap & Min & Max & Position \\
\hline Reg1a & 1.08750 & 0.9000 & 1.1000 & 14 \\
\hline Reg1b & 1.02500 & 0.9000 & 1.1000 & 4 \\
\hline Reg1c & 1.03125 & 0.9000 & 1.1000 & 5 \\
\hline Reg2a & 1.08125 & 0.9000 & 1.1000 & 13 \\
\hline Reg2b & 1.08125 & 0.9000 & 1.1000 & 13 \\
\hline Reg2c & 1.07500 & 0.9000 & 1.1000 & 12 \\
\hline
\end{tabular}

As a final case for the snap shot simulation study, the 34 feeder is simulated with loads projected to grow at a $3 \%$ annual rate for the next 10 years. It was found that given no additional compensation for the next 10 years, the feeder experiences severely depressed voltages at a number of locations. It is also found that the most of the regulators hit their maximum positions at the end of year 10. This suggests that the feeder will need more voltage correction in the future. Some of it could be overcome by adding new reactive support along the feeder length as the loads continue to grow while some of it could be combatted by updating the control mechanism and settings of the voltage regulators. Table 3 lists the regulator tap position at the year 10 with a projected $3 \%$ annual load growth on the feeder load. Table 4 lists additional results of the feeder at the end of 10 years with a sustained annual load buildup of $3 \%$. It can be concluded that the feeder performance deteriorates under the presence of increased load and no additional compensation. The line losses at the end of year 10 equal $574.3 \mathrm{~kW}$. Of course, it is because the feeder has to satisfy a higher load demands which means higher flows across the feeders and the laterals.
Table 3. Regulator Tap Positions at Year 10

\begin{tabular}{|l|l|l|l|l|}
\hline Name & Tap & Min & Max & Position \\
\hline Reg1a & 1.10000 & 0.9000 & 1.1000 & 16 \\
\hline Reg1b & 1.07500 & 0.9000 & 1.1000 & 12 \\
\hline Reg1c & 1.08125 & 0.9000 & 1.1000 & 13 \\
\hline Reg2a & 1.10000 & 0.9000 & 1.1000 & 16 \\
\hline Reg2b & 1.10000 & 0.9000 & 1.1000 & 16 \\
\hline Reg2c & 1.10000 & 0.9000 & 1.1000 & 16 \\
\hline
\end{tabular}

The total load power being met at the end of year 10 is $2341.1 \mathrm{~kW}$ which is much higher than the base case load with full compensation. The substation power factor also falls down to 0.955 which is due to the fact that the reactive power requirement from the source has surged to $906 \mathrm{kVAr}$. The most troubling consequence of the load increase is perhaps the deteriorating voltage profile. The minimum voltage in the feeder drops down to $0.8 \mathrm{p} . \mathrm{u}$ which is totally unacceptable. Hence with no new voltage compensation and an average load growth of $3 \%$ for the next 10 years, the feeder does not meet the necessary requirements of a solved case.

Table 4. Case Results Summary at Year 10

\begin{tabular}{|l|l|}
\hline $\begin{array}{l}\text { Active Power Supplied by } \\
\text { Source }\end{array}$ & $2932 \mathrm{~kW}$ \\
\hline $\begin{array}{l}\text { Reactive Power Supplied by } \\
\text { Source }\end{array}$ & $906 \mathrm{kVAr}$ \\
\hline Source Power Factor & 0.9555 \\
\hline Line Losses & $574.3 \mathrm{~kW}$ \\
\hline Transformer Losses & $23.8 \mathrm{~kW}$ \\
\hline Total Losses & $598.1 \mathrm{~kW}$ \\
\hline Total Load Power & $2341.1 \mathrm{~kW}$ \\
\hline Percent Loss for Circuit & $25.5 \%$ \\
\hline
\end{tabular}

\subsection{Quasi-Static Time Series Simulation}

To evaluate the impact on the voltage regulation equipment under the presence of a time varying PV output and system loading, QSTS simulation tests are performed on the IEEE 34 node test feeder in OpenDSS. Since the feeder is located in Arizona, real residential load profile data is used with a time resolution of 1 hour. Residential load profiles of higher frequency than 1 hour are difficult to obtain and are not always made public by the utilities. Furthermore, it is assumed that all the loads on the feeder experience the same load profile. The time period selected for the load profile is 1 year, from January 1, 2012 to December 31,2012. This takes in to account the seasonal variations in the load with summer season experiencing heavy loads, expectedly, most of which can be attributed to the air conditioning load. Figure 4 
plots the feeder load for the first week of January 2012. Since the collected data is quite large, in order to make visual sense, the load profile for one week is shown here. The feeder load varies as the seasons change with peaks occurring in about mid-summer. The peak feeder load occurs on July 30, 2012 and is equal to $7800 \mathrm{~kW}$. Throughout the year, the load varies from approximately $700 \mathrm{~kW}$ to more than $7500 \mathrm{~kW}$. To illustrate the difference between the amount of irradiance received by a tilted PV cell as opposed to a horizontal surface. Figure 5 shows a plot of GHI, DNI, DHI and GTI (Global Tilted Irradiance) as observed on January 16, 2012.

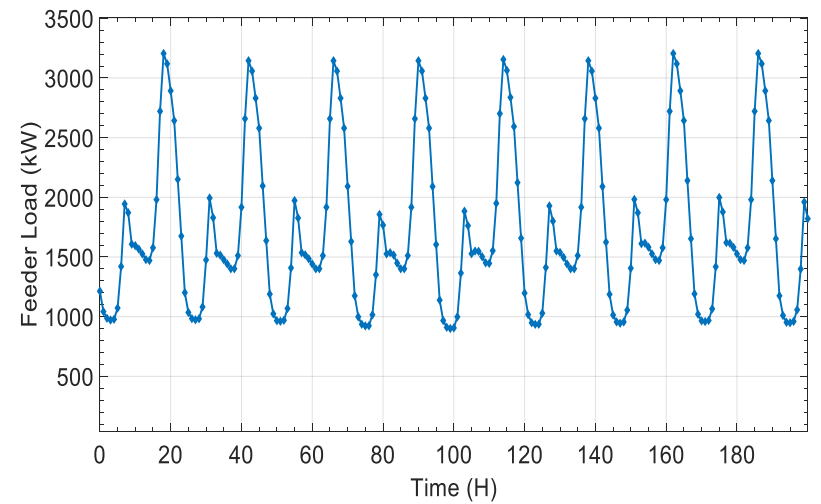

Figure 4. Feeder Load in first week of Jan'12

The effect of the panel tilt angle and the array azimuth is quite apparent in the irradiance profile and if these angles are not accounted for properly, it can lead to wrong conclusions about the operational irregularities in the tap changing devices. . A two-week GTI profile incident on the PV arrays is plotted in Figure 6. To simulate the effect of the PV generation on the action of the tap changers, various degrees of $\mathrm{PV}$ penetration were tested, ranging from $10 \% \mathrm{PV}$ penetration up until $50 \%$ PV penetration. Looking at the annual load profile of the feeder, it is observed that the mean annual load is approximately $2600 \mathrm{~kW}$. The percentage of $\mathrm{PV}$ penetration was defined as the ratio of the PV kWp to the annual mean load on the feeder. PV installations were scattered over the entire feeder with $\mathrm{kWp}$ of each PV installation proportional to the aggregate load at the distribution bus. With this definition a $10 \%$ PV penetration translates approximately to $250 \mathrm{~kW}$ of peak PV output while a $50 \%$ PV penetration yields a peak PV output of roughly $1200 \mathrm{~kW}$. Every PV array is modelled with an inverter whose rated $\mathrm{kVA}$ equals the $\mathrm{kWp}$ of the PV array at unity power factor.

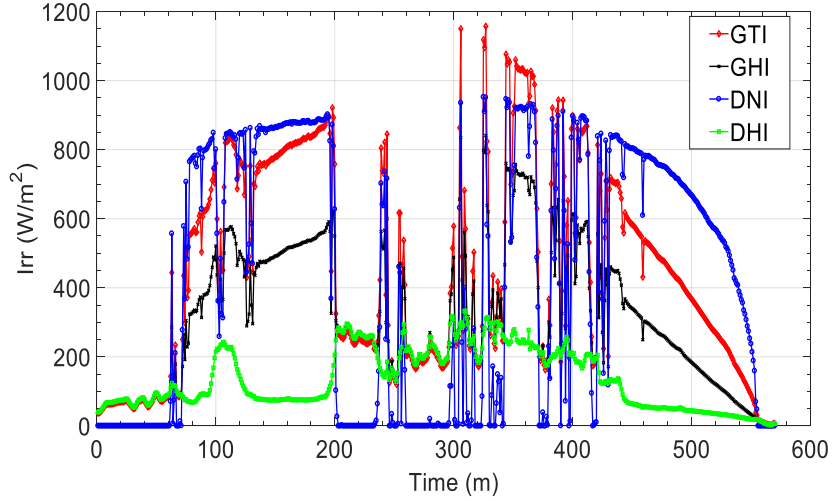

Figure 5. Different Irradiance Profiles

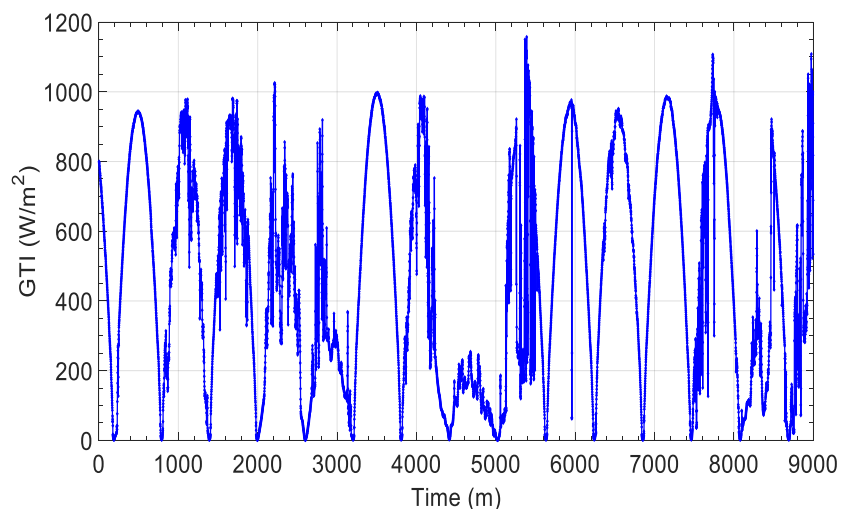

Figure 6. Tilted Irradiance for two weeks in January, 2012.

Figure 7 and Figure 8 show the active power flowing through Regulator 1 and the corresponding changes in the tap position. The plots are indicative of feeder conditions from January 1 to January 16, 2012. As the day begins on January 1, 2012 the PV output begins to ramp up which is reflected as a drop in active power flowing through the regulator in proportional to the degree of PV penetration on the feeder. Since the variabilities in the power through regulator are at a minimum, thus indicating a smooth ramp of the PV output, changes in tap positions are not significant. However, as the week progresses, the stochastic nature of the PV generation, introduces random fluctuations in the active power through the regulator and this leads to more tap operations in order to maintain the voltage in a certain defined band. It is also clear that at low PV penetration, the transformer tap does not vary much as opposed to high PV penetration. At high penetration, a small variability in the $\mathrm{PV}$ generation can cause the transformer tap to rapidly change position. It can also be observed that at high PV penetrations, reverse power flow can also take place across the regulator terminals. 


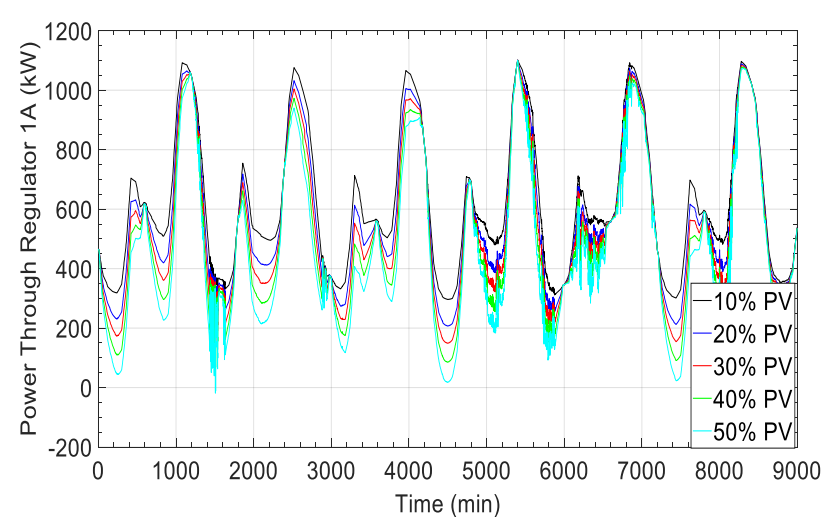

Figure 7. Active Power through Reg. 1A

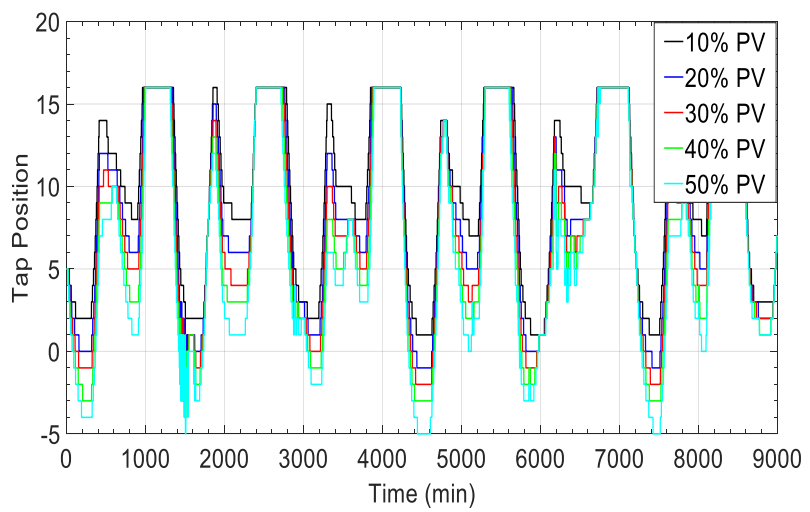

Figure 8. Changes in Tap Position of Reg. 1A

From Figure 7, at PV penetration of $50 \%$ reverse power flow happens on roughly on January 3, 2012 for a brief period of time. On this day the tilted irradiance hits the peak of roughly $1 \mathrm{~kW} / \mathrm{m}^{2}$ which explains the high PV output during this particular time of this day of the year. The feeder at this point in time is relatively lightly loaded with a total system load of roughly 1400 $\mathrm{kW}$ which is less than the base active power load of the system. Both the regulators exhibit similar behavior under high PV penetration, although the number of tap change operations differs. The location of regulator 2 makes it vulnerable to severely depressed voltages under peak load conditions. That explains the higher number of tap change operations in regulator 2 as compared to regulator 1 .

To quantify the number of annual tap changes with a load profile given in Figure 4 and the tilted irradiance profile shown in Figure 6, the IEEE 34 node feeder is subjected to time series simulations with varying degree of PV penetration. The maximum increase in the number of annual tap operations was found to occur in regulator $2 \mathrm{~A}$, with 33580 tap operations recorded under a PV penetration of $50 \%$. In the absence of any PV generation, the number of annual tap operations in regulator $2 \mathrm{~A}$ were found to be 9855 . This represents an approximate increase of 3.5 times in the number of annual tap operations. Given a rough estimate of the average life of a load tap changer to be 500,000 operations, a $50 \% \mathrm{PV}$ penetration on the feeder can shorten the life of the voltage regulator from 50 years to roughly about 15 years. Figure 9 shows the annual tap operations of the individual phases of the two regulators in the IEEE 34 node feeder as a function of $\mathrm{PV}$ penetration.

The voltage profile of Bus 890 is shown in Figure 9. This bus is located at approximately 55 miles from the substation transformer and often exhibits depressed voltages, even with the compensation provided by the capacitors and the voltage regulators. The power to this bus is fed from the secondary of the in-line transformer which steps down the line voltage $24.9 \mathrm{kV}$ to $4.16 \mathrm{kV}$. The rapid fluctuations in the voltage are a results of erratic power flow through the transformer. The percentage change in the voltage rise and drop increases with the increase in the PV penetration on the feeder. For a $50 \%$ PV penetration, the maximum rise and drop in the voltage exceeds $1.5 \%$ during times when the intermittency of the tilt irradiance increases. On an average the changes in the voltage are in the order of $0.5 \%-1 \%$. Such rapid change in the voltage can induce flicker problems especially in conditions of high PV penetration and an increased variability of the solar irradiance. Given the upper threshold for the visibility of flicker to be $0.7 \%$ for 1-minute interval, it can be concluded that flicker could be an issue under high penetration of photovoltaic power.

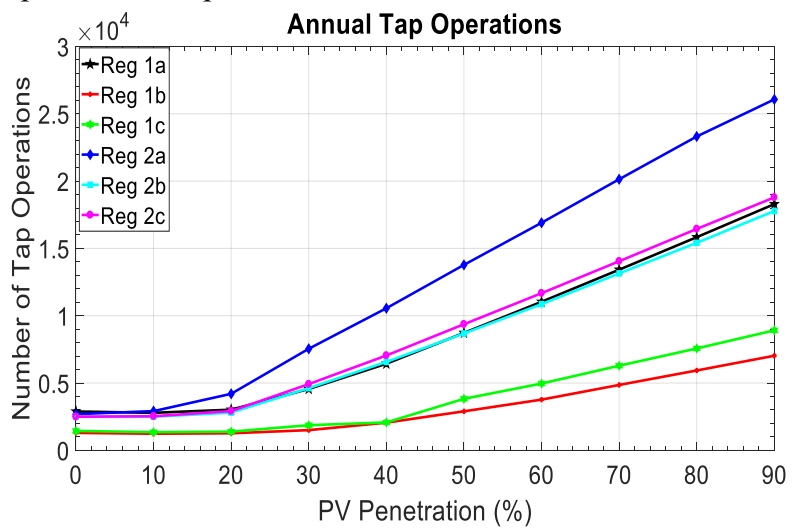

Figure 9. Tap operation with and without PV

\subsection{QSTS- Monte Carlo Simulations}

In order to capture the effect of the varying roof tilt angle and PV array azimuths, the IEEE 34 node feeder was subjected to the MC simulations. The de-rating of the voltage regulating equipment due to the intermittency associated with the PV generation is a deterministic problem, since a fixed set of parameter values or inputs will always lead to the same output. 
Unlike stochastic models, the model does not have inherit randomness.

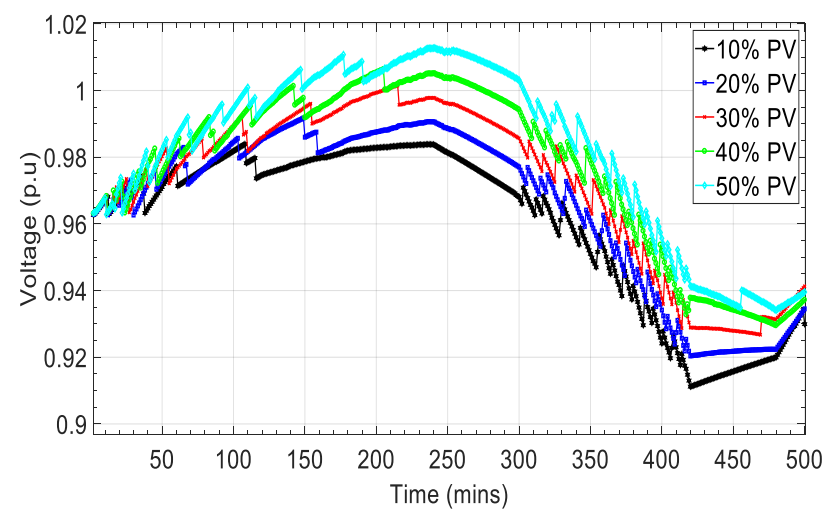

Figure 10. Voltage at Bus 890 (far end of the feeder) on January 1, 2012

The randomness or uncertainty however lies in the set of input parameter values that the model can take. These values include a changing load profile with time, a time-dependent PV generation, different PV array azimuths and tilt angles. To quantify the impact of the randomness associated with the input parameters, Monte Carlo method is used to iteratively evaluate a deterministic model, which consists of a distribution feeder with and without PV penetrations, using random numbers as inputs. The input parameters considered include load profile, PV array azimuth angles and PV tilt angles. In order to characterize the randomness of the load profiles, each of the scaled 8760 load values was multiplied by a factor sampled randomly from a Normal distribution with a mean of 1 and a standard deviation of 0.035 . As a first approximation and because of the lack of data at the present, the PV azimuth angle and the tilt angles were sampled from a uniform distribution. Since the feeder is located in the Northern Hemisphere, the PV azimuths were sampled in the interval of [ 90 degrees (E), 270 degrees (W)] with the optimum occurring at 180 degrees (S). The array azimuth is the angle clockwise from true north describing the direction that the array faces. The default value for a South facing array is 180 degrees and 0 degrees for a north facing array. For the tilt angles, since the most common roof pitch in the United States is anywhere in between 4/12 and $9 / 12$ which corresponds to the range 18.43 degrees to 26.87 degrees with the horizontal surface, the tilt angles were sampled from a uniform distribution in the interval [25 degrees, 35 degrees]. Based on the distance of the buses from the substation transformer and from each other, the IEEE 34 node test feeder is divided into nine zones. Buses or nodes which are within roughly 2-3 miles of each other are grouped into one zone which are then assigned same values for the PV array azimuth and the tilt angle. As such for every Monte Carlo run, the effect of nine different PV array azimuths and tilt angles is simulated. Since the total number of MC runs is 200 for every feeder condition, 1800 different irradiance profiles are created and tested. The feeder is simulated with $0 \%$ PV up until 90\% PV penetration with intermediate

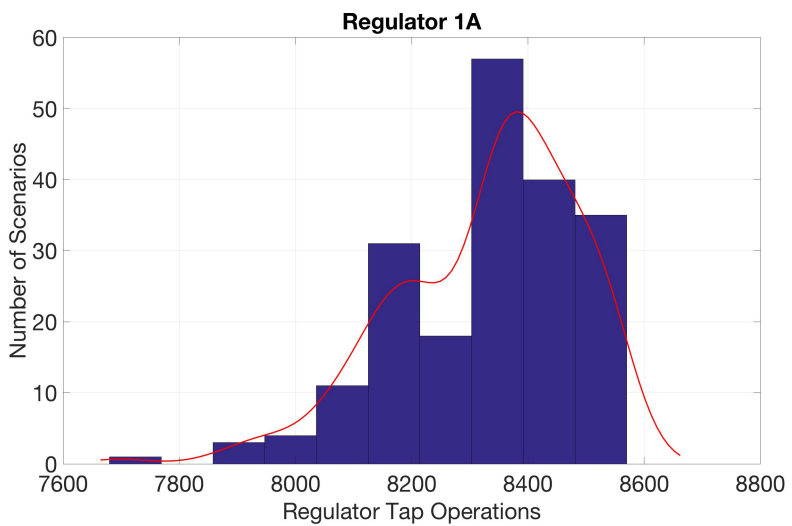

Figure 11. Distribution of Tap Operations of Reg $1 A$ at $0 \%$ PV

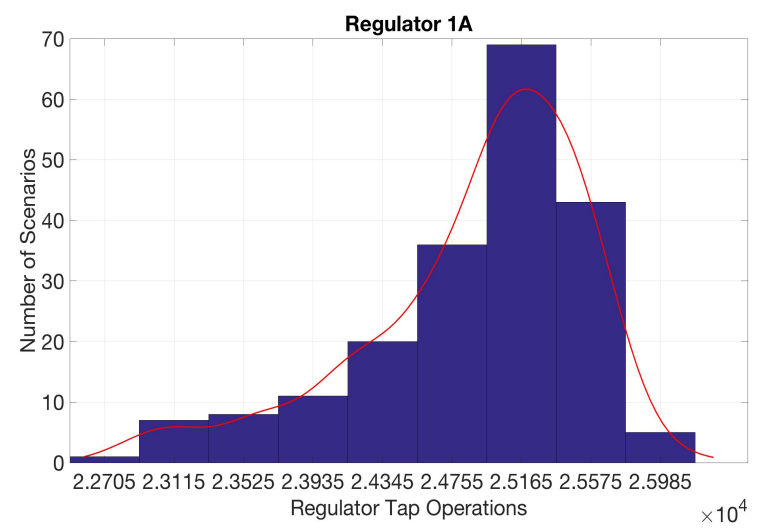

Figure 12. Distribution of Tap Operations of Reg $1 \mathrm{~A}$ at $90 \% \mathrm{PV}$

steps of $30 \%, 50 \%$ and $70 \%$ PV penetration. Due to the lack of space, the results for regulator $1 \mathrm{~A}$ at $0 \% \mathrm{PV}$ and $90 \%$ PV penetration are shown here. The tap operation distributions as shown in Figure 10 and Figure 11 can be used to derive a probabilistic estimate about the de-rating of the voltage regulation equipment. By making use of dependent discrete convolution, it is possible to combine the distribution of tap operations with and without PV on the feeder. In order to quantify the impact of PV on the operational lifetime of the electro-mechanical devices in some probabilistic sense, the resulting distribution could then be compared with the average lifetime of the device, which again is better represented as a distribution of numbers rather than a single number. This section is an ongoing work which will be expanded in much more detail in the future. 
To mitigate the deleterious effects of the solar PV generation on the operational lifetime of the electromechanical devices on distribution feeders, a model predictive controlled, matrix-converter-basedpower quality compensator (MPC-MC-PQC) is proposed with an operational lifetime of four decades by not using electrolytic capacitors. The proposed PQC is optimized for distribution systems, designed to provide reactive and harmonic power mitigation at the source rather than upstream in the network. This is a work in progress and will be addressed explicitly in future publications of this work.

\section{Conclusions}

The article presents the impact of the solar PV intermittency with and without a sustained load buildup on the operation of the electro-mechanical devices which are designed for regulating the voltage in the distribution networks. Snap shot power flow simulations as well as quasi-static time series simulations are performed on an actual residential radial feeder with a yearlong real-world solar data of time resolution 1 minute and the residential load profiles with time resolution of 1 hour. For the solar irradiance incident on the solar PV array, the effect of the tilt angle of the array and the array azimuth is considered. The simulations utilize full three phase models of the circuit elements which is important for distribution system analysis because of the inherent imbalance present in the phases. Based on the snap shot simulation study, it was observed that the IEEE 34 node feeder is not provided with adequate voltage compensation. Certain buses on the feeder, in particular Bus 890, exhibit depressed voltages, less than 0.95 p.u. With an annual load growth of $3 \%$ for the next years, it was found that in the absence of any new additional compensation, the feeder performance will drop precipitously over the next decade with unacceptable voltages at many buses. Under sustained load buildup, the feeder would require substantial voltage support to combat the increasing load.

Time series simulations reveal how the variability in the PV output affects the operational activity of the regulator tap changers. It is estimated that an increased PV penetration on the feeder can cause some regulators to operate roughly four times more which can shorten the life span of such devices by as much as $70 \%$. This represents significant wear and tear of the regulators and needs to be considered before any sort of distribution generation can be allocated on the feeder. For the feeder voltage, it is seen that an increased PV penetration induces erratic changes in the voltage profile, which resembles a saw-tooth structure with higher voltages for high PV penetration.
The problem of voltage rise in the presence of PV under conditions of light load needs to be combatted in order to keep the voltage within limits.

The future work in this direction will involve expanding the scope of the stochastic simulations to include the operational activity of the capacitor banks as well as the substation LTC transformer in addition to that of the voltage regulators. The use of stochastic simulations is necessary to derive a probabilistic estimate of the derating of the voltage regulating equipment.

\section{Acknowledgement}

This publication was made possible by NPRP grant \# 9-204-2-103 from the Qatar National Research Fund (a member of Qatar Foundation). The statements made herein are solely the responsibility of the authors.

\section{References}

[1] Liu, Xiaohu, et al. "Coordinated control of distributed energy storage system with tap changer transformers for voltage rise mitigation under high photovoltaic penetration." IEEE Transactions on Smart Grid 3.2 (2012): 897-906.

[2] Liu, Y., et al. "Distribution system voltage performance analysis for high-penetration PV." Energy 2030 Conference, 2008. ENERGY 2008. IEEE. IEEE, 2008.

[3] Ari, G. K., and Yahia Baghzouz. "Impact of high PV penetration on voltage regulation in electrical distribution systems." Clean Electrical Power (ICCEP), 2011 International Conference on. IEEE, 2011.

[4] Yan, Ruifeng, Brandon Marais, and Tapan Kumar Saha. "Impacts of residential photovoltaic power fluctuation on on-load tap changer operation and a solution using DSTATCOM." Electric Power Systems Research 111 (2014): 185-193.

[5] Lave, Matthew, Matthew J. Reno, and Robert J. Broderick. "Characterizing local high-frequency solar variability and its impact to distribution studies." Solar Energy 118 (2015): 327-337.

[6] Bank, Jason, and Barry Mather. "Analysis of the impacts of distribution connected PV using high-speed datasets." Green Technologies Conference, 2013 IEEE. IEEE, 2013.

[7] Hanley, Charles, et al. "Technology development needs for integrated grid-connected PV systems and electric

[8] Agalgaonkar, Yashodhan P., Bikash C. Pal, and Rabih A. Jabr. "Distribution voltage control considering the impact of PV generation on tap changers and autonomous regulators." IEEE Transactions on Power Systems 29.1 (2014): 182-192.

[9] Gueymard, Christian A. "From global horizontal to global tilted irradiance: how accurate are solar energy engineering predictions in practice?." American Solar Energy Society(2008). 